\title{
Correlation between root respiration and the levels of biomass and glycyrrhizic acid in Glycyrrhiza uralensis
}

\author{
WENLAN LIU, ZHIRONG SUN, JIXU QU, CHUNNING YANG, XIAOMIN ZHANG and XINXIN WEI \\ School of Chinese Materia Medica, Beijing University of Chinese Medicine, Beijing 100029, P.R. China
}

Received October 18, 2015; Accepted December 1, 2016

DOI: $10.3892 /$ etm.2017.4723

\begin{abstract}
The aim of the present study was to investigate the correlation between root respiration and the levels of biomass and glycyrrhizic acid in Glycyrrhiza uralensis. Root respiration was determined using a biological oxygen analyzer. Respiration-related enzymes including glucose-6-phosphate dehydrogenase plus 6-phosphogluconate dehydrogenase, phosphohexose isomerase and succinate dehydrogenase, and respiratory pathways were evaluated. Biomass was determined by a drying-weighing method. In addition, the percentage of glycyrrhizic acid was detected using high-performance liquid chromatography. The association between root respiration and the levels of biomass and glycyrrhizic acid was investigated. The glycolysis pathway (EMP), tricarboxylic acid cycle (TCA) and pentose phosphate (PPP) pathway acted concurrently in the roots of $G$. uralensis. Grey correlation analysis showed that TCA had the strongest correlation (correlation coefficient, 0.8003) with biomass. Starch and acetyl coenzyme A had the closest association with above-ground biomass, while soluble sugar correlated less strongly with above-ground biomass. Grey correlation analysis between biochemical pathways and the intermediates showed that pyruvic acid had the strongest correlation with EMP, while acetyl coenzyme A correlated most strongly with TCA. Among the intermediates and pathways, pyruvic acid and EMP exhibited the greatest correlation with glycyrrhizic acid, while acetyl coenzyme A and TCA correlated with glycyrrhizic acid less closely. The results of this study may aid the cultivation of G. uralensis. However, these results require verification in further studies.
\end{abstract}

\section{Introduction}

Glycyrrhiza uralensis Fisch. (G. uralensis), a medicinal herb, has been frequently used in the food processing and daily

Correspondence to: Professor Zhirong Sun, School of Chinese Materia Medica, Beijing University of Chinese Medicine, 6 South Road, Beijing 100029, P.R. China

E-mail: zrs67@126.com

Key words: Glycyrrhiza uralensis, respiration, glycyrrhizic acid, biomass, gray correlation chemical industries. However, wild G. uralensis resources have been severely destroyed, and in some areas, are at risk of extinction (1). In recent years, artificial cultivation of G. uralensis has been considered as an alternative to the wild resources. However, a number of problems have encountered regarding its artificial cultivation, including uncertainty regarding the mechanism by which glycyrrhizic acid, the active component of $G$. uralensis, is affected by the ecological environment, poor root quality and low content of glycyrrhizic acid. Extensive studies have been performed to investigate the effects of nutrients (2), seeding density (3) and water (4) on the content of glycyrrhizic acid in cultivated G. uralensis. However, the optimum conditions for increasing the production of glycyrrhizic acid have not been identified.

Root respiration plays an important role in the primary productivity of plants (5). Sugar metabolism, including the tricarboxylic acid cycle (TCA), pentose phosphate pathway (PPP) and glycolysis pathway (EMP), is the primary metabolism associated with respiration (6-8). In respiration, a variety of intermediate products are generated through the decomposition of glucose, which provide energy and substrates for primary metabolites such as lipids, proteins and nucleic acids, and secondary metabolites such as terpenoids and flavonoids. When the respiratory intensity and pathway are modified, the number and types of metabolic intermediates are affected, which causes a series of changes to the metabolism of other materials and ultimately affects the growth and development of plants (9). In our previous study (10), it was found that the root respiration rate was correlated with the levels of biomass and glycyrrhizic acid in G. uralensis. However, the effects of environmental and metabolic pathways on the accumulation of biomass and glycyrrhizic acid in G. uralensis have not been evaluated.

In the present study, the fluctuations of root respiration were analyzed, and the correlations between root respiration and the levels of biomass and glycyrrhizic acid in $G$. uralensis were investigated. The results of this study may promote the sustainable use of $G$. uralensis resources and the cultivation of G. uralensis.

\section{Materials and methods}

Samples. G. uralensis seeds and seedlings were grown in Hangjingqi (Inner Mongolia, China). The plants were cultivated in April 2014 in a growth chamber at the Beijing 
University of Chinese Medicine (Beijing, China; $39^{\circ} 55^{\prime} \mathrm{N}$, $116^{\circ} 28^{\prime} \mathrm{E}$, at an altitude of $\left.54.7 \mathrm{~m}\right)$. The plants were cultivated at an average temperature of $14.1^{\circ} \mathrm{C}$ and an annual rainfall of $500 \mathrm{~mm}$.

The G. uralensis was grown in a polyvinyl chloride (PVC) tube, which was $5 \mathrm{~cm}$ above the ground. The tube was filled with sandy loam, containing organic matter $(0.279 \%)$, alkali-hydrolyzable nitrogen $(35.85 \mathrm{mg} / \mathrm{kg})$, soil-available phosphorus $(2.9 \mathrm{mg} / \mathrm{kg})$, soil-available potassium $(85.11 \mathrm{mg} / \mathrm{kg})$ and $\mathrm{CaCO}_{3}(2.69 \%)$. The $\mathrm{pH}$ value of the sandy loam was maintained at 7.91. The bottom of the tube was sealed with gauze. Seeds were soaked in $\mathrm{H}_{2} \mathrm{SO}_{4}$ (98\%) for $45 \mathrm{~min}$, and soaked for a further $12 \mathrm{~h}$ after disinfection with $\mathrm{KMnO}_{4}(0.2 \%)$. Six G. uralensis plants were cultivated in one PVC tube. No fertilizer was given to the plants. The seeding and transplantation of the G. uralensis were conducted on May 10. Plants were watered with self-prepared improved Hoagland nutrient solution with a $\mathrm{pH}$ value of 7.4 from April to November on the 15th day of each month. The Hoagland nutrient solution was administered once every 15 days over the same time period. The soil moisture status was kept at $60 \%$.

Measurement of the rate of respiration. To determine respiration rate, the root was washed with distilled water and wrapped with wet gauze. The root was soaked in $\mathrm{H}_{2} \mathrm{O}_{2}(3 \%)$ solution after washing twice with neutral detergent, in order to minimize the effect of root microorganisms. The rate of respiration was measured according to the previously described method (11). In brief, EMP, TCA and PPP pathways were inhibited using $0.2 \mathrm{M} \mathrm{NaF}$ solution ( $\mathrm{pH}$ 7.4), $0.2 \mathrm{M}$ propanedioic acid solution (pH 7.4) and trisodium phosphate solution ( $\mathrm{pH}$ 7.4), respectively. The root was then placed in a Yaxin-1151 Biological Oxygen analyzer (Yaxinliyi Science \& Technology Co., Ltd. Beijing, China), and the net photosynthetic oxygen evolution rate was measured at $25^{\circ} \mathrm{C}$.

Determination of substrate and intermediate products. The levels of soluble sugar and starch were measured by a colorimetric method using anthrone as previously described (12). Pyruvic acid (also known as pyroracemic acid) was detected using an improved 2,4-dinitrophenylhydrazine colorimetric method (13). Levels of acetyl coenzyme A was determined using a commercial plant acetyl coenzyme A (A-CoA) ELISA kit (cat no. GTX500030; Huaxia Yuanyang Scientific Co., Ltd., Beijing, China), according to the manufacturer's instructions.

Determination of respiration-related enzyme activity. The activity of phosphohexose isomerase (PHI) was assayed according to the method described by Brown and Wray (14). Fresh tissues $(0.5 \mathrm{~g})$ were homogenized in $1.5 \mathrm{ml}$ cold Tris- $\mathrm{HCl}$ (pH 7.4), followed by centrifugation at $4,689.8 \times \mathrm{g}$ for $30 \mathrm{~min}$ at $4^{\circ} \mathrm{C}$. The supernatant was used for analysis of enzyme activity. The enzyme activity was calculated according to the following formula: (A - 0.1374) x $0.872 /(0.2886 \mathrm{x}$ fresh weight $\mathrm{x} t)$, where $\mathrm{t}$ represents the time point of determination and $\mathrm{A}$ represents absorption value.

The total activity of 6-phosphogluconate dehydrogenase (6-PGDH) and glucose-6-phosphate dehydrogenase (G-6-PDH) was determined using the method of Brown and Wray (14). Fresh plant tissues $(0.5 \mathrm{~g})$ were homogenized in
$1.5 \mathrm{ml}$ cold Tris- $\mathrm{HCl}(\mathrm{pH} 7.4)$ and centrifuged at $4,689.8 \mathrm{x} \mathrm{g}$ for $30 \mathrm{~min}$ at $4^{\circ} \mathrm{C}$. The mixture contained $1.35 \mathrm{ml} 0.15 \mathrm{mmol} / 1$ Tris- $\mathrm{HCl}$ ( $\mathrm{pH}$ 7.4, $5 \mathrm{mM}$ fructose-6-phosphate, $5 \mathrm{mM} \mathrm{MgCl} 2$ and $5 \mathrm{mM}$ NADP) and $0.15 \mathrm{ml}$ enzyme-containing plant extract. The activity was measured at $340 \mathrm{~nm}$. Enzyme activity was expressed in units of $\mu \mathrm{mol} \mathrm{NADP} / \mathrm{min} / \mathrm{g}$ fresh weight. The enzyme activity was calculated as follows: Enzyme activity $=1.5 \mathrm{~F} \times \Delta \mathrm{A} /(0.1 \mathrm{x}$ fresh weight $\mathrm{x} \mathrm{t})$, where $\mathrm{F}=1,607.7$, $\Delta \mathrm{A}$ stands for changes of absorption and $\mathrm{t}$ represents the time point.

The activity of succinate dehydrogenase (SDH) was determined as previously described (7). The absorbance was determined at $600 \mathrm{~nm}$. One unit of enzyme activity was defined as an increase of $1 \mathrm{in}$ absorbance/min/g.

Determination of biomass and glycyrrhizic acid of G. uralensis. Representative plants were selected, and the leaf number, plant height, above-ground biomass and below-ground biomass were determined. The glycyrrhizic acid content was then analyzed using high performance liquid chromatography (HPLC), as previously described (15). A chromatographic column (C18, 250x4.6 mm, $5 \mu \mathrm{m}$; Agilent Technologies Inc., Santa Clara, CA, USA) was used for the analysis.

Statistical analysis. Following normalization of the data, gray relational analysis (16) between root respiration and the levels of biomass and glycyrrhizic acid in G. uralensis was conducted using Data Processing System (DPS) 7.05 software (Microsoft Corporation, Redmond, WA, USA). One way analysis of variance followed by a least significant difference test was used to analyze differences between groups and calculate P-values. Data analysis was performed using SPSS 17.0 software and $\mathrm{P}<0.05$ was considered to indicate a statistically significant difference.

\section{Results}

Dynamic changes of the respiration rate. The total respiration rate of the licorice root system gradually decreased from August to October. The total respiration rate was at the highest level in early August, and decreased to a minimum value in late October (Fig. 1). No significant difference was observed in the total respiration rate between the designated months $(\mathrm{P}>0.05)$.

As shown in Fig. 2, EMP, TCA and PPP pathways acted concurrently at all the investigated times, while the proportion of each pathway varied over time. The proportion of the EMP pathway was a maximum at early August, when it comprised $\sim 36.54 \%$ of the total respiration rate. It then decreased rapidly, reaching a minimum in early September, at $24.52 \%$ of the total respiration rate and then rapidly increased by late September, when it comprised $\sim 32.51 \%$ of the total respiration rate. The proportion of the TCA pathway increased during August, reaching a maximum of $\sim 40.66 \%$ of the total respiration rate at late August; it then declined, reaching a minimum of $\sim 33.84 \%$ of the total respiration rate at late October $(\mathrm{P}<0.05)$. The proportion of the PPP pathway increased to a maximum of $31.98 \%$ of the total respiration rate in early September; its minimum was in early August, which was $\sim 0.69$-fold lower than that of early September. 


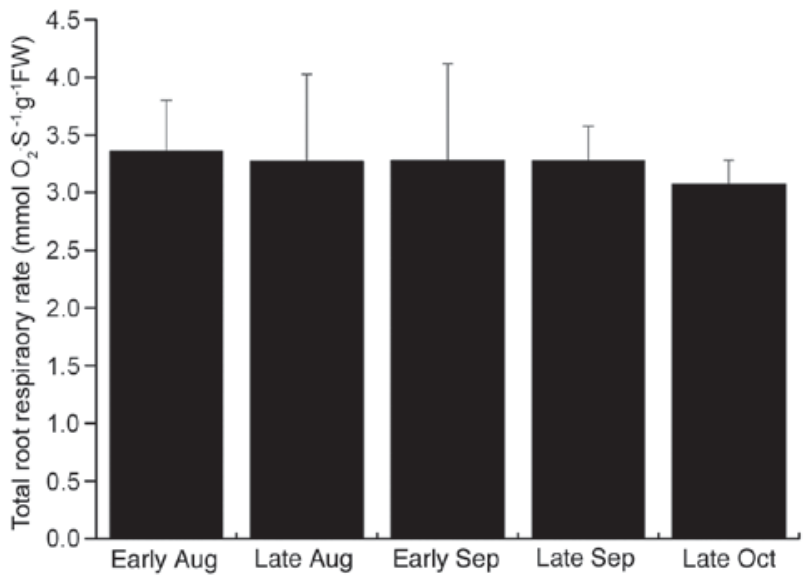

Figure 1. Dynamic changes of the total respiration rate in the root of Glycyrrhiza uralensis. The data were presented as mean \pm standard deviation from at least three experiments. FW, fresh weight.

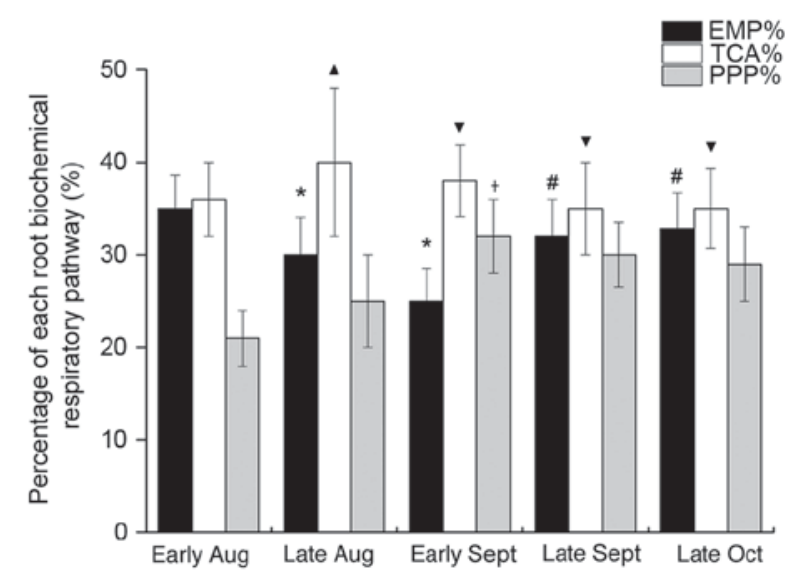

Figure 2. Dynamic changes of the percentage of each respiratory pathway in the root of Glycyrrhiza uralensis. The data were presented as mean \pm standard deviation from at least three experiments. ${ }^{*} \mathrm{P}<0.05$, compared with early August. " $\mathrm{P}<0.05$, compared with early August. ${ }^{\dagger} \mathrm{P}<0.05$, compared with early August. ${ }^{\Delta} \mathrm{P}<0.05$, compared with early August. ${ }^{\mathbf{}} \mathrm{P}<0.05$, compared with late August. EMP, glycolysis pathway; TCA, tricarboxylic acid cycle; PPP, pentose phosphate pathway.

Determination of substrate products. As presented in Fig. 3, the proportions of soluble sugar and starch were the lowest in early August. The proportions then increased rapidly, reaching a maximum in early September, followed by a decline. Soluble sugar and starch rapidly accumulated during the period from late August to early September. The content of soluble sugar in late September was 6.9-fold higher than that of late August $(\mathrm{P}<0.05)$ and the content of starch in late September was 1.8 -fold higher than that of late August $(\mathrm{P}<0.05)$. The contents of soluble sugar and starch exhibited only slight changes from early September to late September $(P>0.05)$. The content of soluble sugar in late October was $54.4 \%$ of that in late September $(\mathrm{P}<0.05)$ and the starch content in late October was $59.6 \%$ of that in late September $(\mathrm{P}<0.05)$.

Determination of intermediate products. As indicated in Fig. 4, significant differences were observed in the content of pyruvic acid among the various measurement times. The content of pyruvic acid initially increased and then decreased slightly.

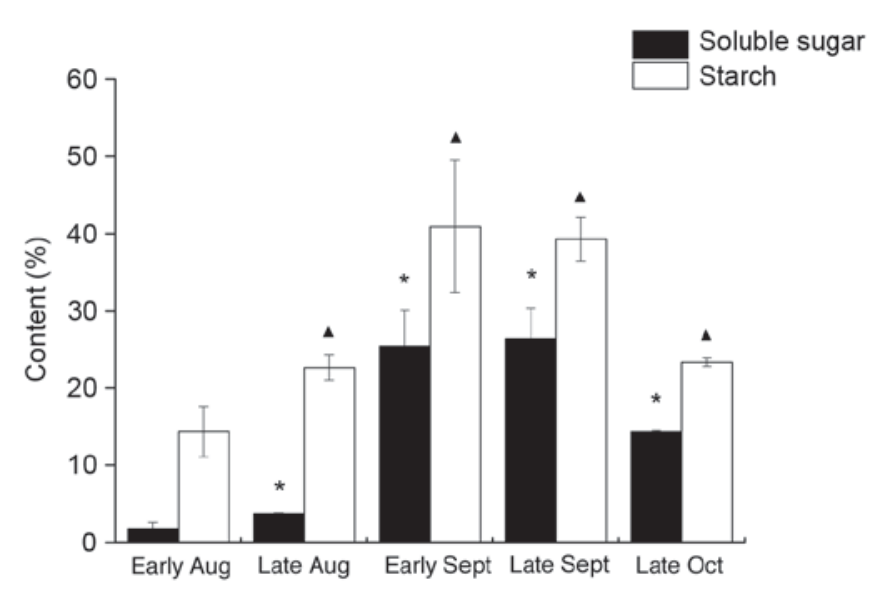

Figure 3. Dynamic changes of soluble sugar and starch in the root of Glycyrrhiza uralensis. The data were presented as mean \pm standard deviation from at least three experiments. ${ }^{~} \mathrm{P}<0.05$, compared with early August; ${ }^{\Delta} \mathrm{P}<0.05$, compared with early August.

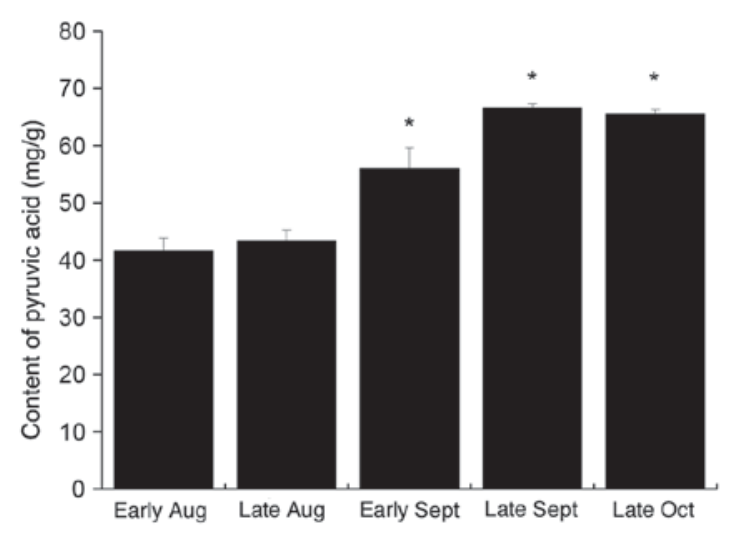

Figure 4. Dynamic changes of pyruvic acid content in the root of Glycyrrhiza uralensis. The data were presented as mean \pm standard deviation from at least three experiments. ${ }^{*} \mathrm{P}<0.05$, compared with early August.

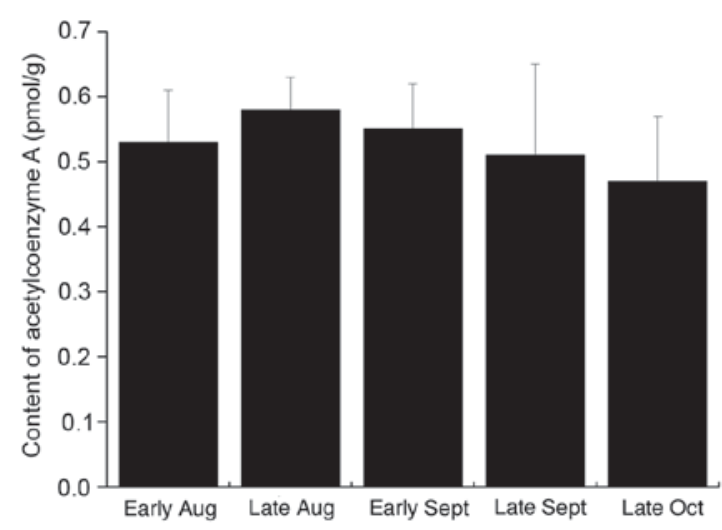

Figure 5. Dynamic changes of acetyl coenzyme A content in the root of Glycyrrhiza uralensis. The data were presented as mean \pm standard deviation from at least three experiments.

Prior to late September, the content of pyruvic acid rapidly accumulated and reached a maximum of $66.62 \mathrm{mg} / \mathrm{g}$ in late September, which was $\sim 1$.6-fold higher than the content of early August $(\mathrm{P}<0.05)$. The pyruvic acid content was slightly lower in late October than in late September. As shown in Fig. 5, the 


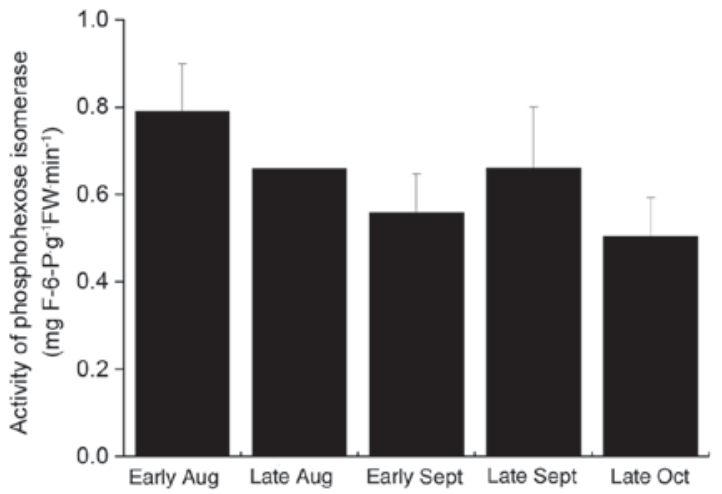

Figure 6. Dynamic changes of the activity of phosphohexose isomerase in the root of Glycyrrhiza uralensis. The data were presented as mean \pm standard deviation from at least three experiments. FW, fresh weight; F-6-P, fructose-6-phosphate.

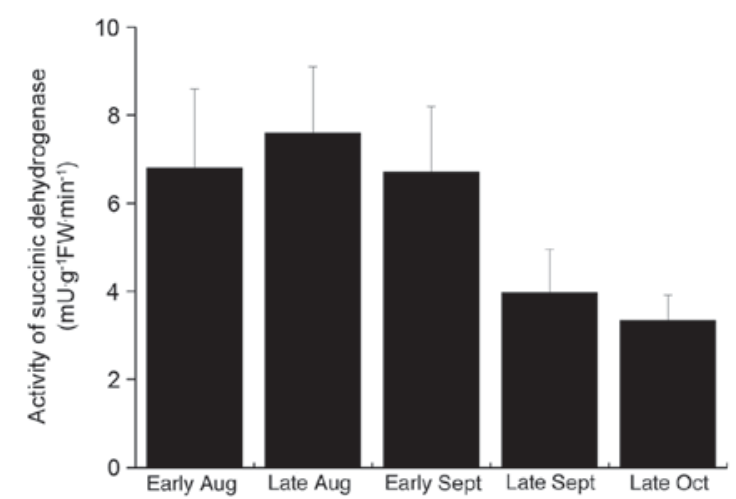

Figure 7. Dynamic changes of the activity of succinic dehydrogenase in the root of Glycyrrhiza uralensis. The data were presented as mean \pm standard deviation from at least three experiments. FW, fresh weight.

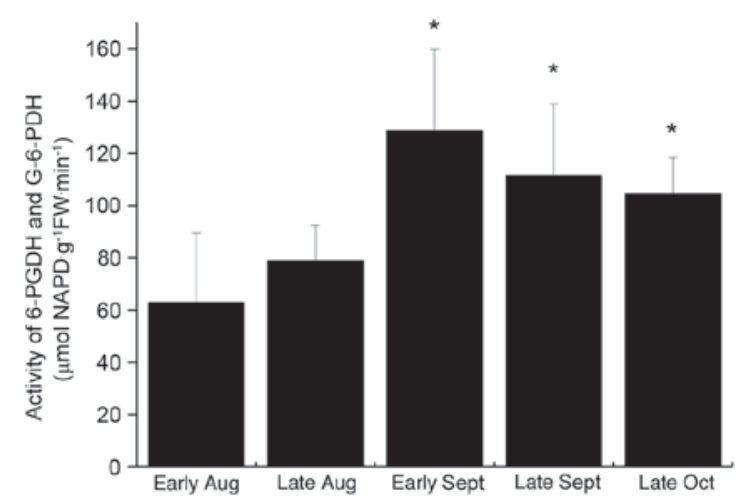

Figure 8. Dynamic changes of the activities of G-6-PDH and 6-PGDH in the root of Glycyrrhiza uralensis. The data were presented as mean \pm standard deviation from at least three experiments. ${ }^{*} \mathrm{P}<0.05$, compared with early August. G-6-PDH, glucose-6-phosphate dehydrogenase; 6-PGDH, 6-phosphogluconate dehydrogenase.

content of acetyl coenzyme A reached a maximal value in late August, while the lowest value was observed in late October and comprised $\sim 85.7 \%$ of that in late August. However, there were no significant differences in the values of acetyl coenzyme $\mathrm{A}$ at each time-point.
Determination of respiration-related enzyme activity. The activity of PHI exhibited a similar trend to the EMP pathway. After reaching a low level in late September, PHI activity increased slightly and then decreased again (Fig. 6). The activity of SDH increased slightly initially, reaching a maximum in late August. Subsequently, it began to decrease and reached a minimum in late October (Fig. 7). The total activity of 6-PGDH and G-6-PDH exhibited a similar trend to the proportion of the PPP pathway, exhibiting an initial increase between early August and early September $(\mathrm{P}<0.05)$ followed by a gradual decline (Fig. 8).

Determination of the biomass of G. uralensis. As presented in Table I, significant differences were observed among above- and below-ground biomass weights $(\mathrm{P}<0.01)$. The above- and below-ground biomass weight gradually increased from August to October. The accumulation of above-ground biomass was uniform, but the below-ground biomass increased most rapidly in late October, when it was $\sim 4.07$-fold higher than that in early September.

Dynamics of glycyrrhizic acid levels. As illustrated in Fig. 9, the glycyrrhizic acid levels significantly increased between early August and October. A rapid accumulation occurred from early August to late September. The content of glycyrrhizic acid in late September was 6.64-fold higher than that of early August $(\mathrm{P}<0.05)$. The content of glycyrrhizic acid increased slightly from late September to late October, though this increase was not significant.

Grey correlation analysis between root respiration and the levels of biomass in G. uralensis. As shown in Table II, the three biochemical pathways were correlated with the aboveand below-ground biomass, and the correlation with the above-ground biomass was greater than that of below-ground biomass. The above-ground biomass was most closely correlated with TCA, followed by PPP and EMP. The below-ground biomass was most closely correlated with EMP, followed by PPP and TCA, respectively.

The contents of respiratory substrates and intermediates were correlated with the above- and below-ground biomass (Table III). The above-ground biomass was closely correlated with starch, followed by acetyl coenzyme A, soluble sugar and pyruvic acid, respectively. In addition, the below-ground biomass was closely correlated with pyruvic acid, followed by acetyl coenzyme A, soluble sugar and starch, respectively.

Table IV summarizes the correlation between biochemical pathways and the content of intermediates in G. uralensis. The results indicated that TCA was closely correlated with the acetyl coenzyme A content. In addition, EMP was closely correlated with the pyruvic acid content.

Correlation between root respiration and the accumulation of glycyrrhizic acid. The biochemical pathways were correlated with the levels of glycyrrhizic acid. EMP showed the closet correlation with the accumulation of glycyrrhizic acid, followed by the PPP and TCA pathways (Table V). As shown in Table VI, the intermediates correlated with the glycyrrhizic acid content. 
Table I. Dynamic changes of biomass weight $(\mathrm{g})$ in Glycyrrhiza uralensis.

\begin{tabular}{|c|c|c|c|c|c|}
\hline Biomass & Early August & Late August & Early September & Late September & Late October \\
\hline Above-ground & $0.33 \pm 0.03$ & $0.54 \pm 0.09$ & $0.76 \pm 0.11$ & $0.91 \pm 0.32$ & $1.03 \pm 0.32$ \\
\hline Below-ground & $0.11 \pm 0.02^{\mathrm{a}}$ & $0.30 \pm 0.05^{\mathrm{a}}$ & $0.73 \pm 0.43$ & $1.24 \pm 0.26^{\mathrm{a}}$ & $2.96 \pm 0.75^{\mathrm{a}}$ \\
\hline
\end{tabular}

${ }^{\mathrm{a}} \mathrm{P}<0.05$ vs. above-ground biomass.

Table II. Correlation coefficients determined by grey correlation analysis between biochemical pathways and the levels of biomass in Glycyrrhiza uralensis.

\begin{tabular}{lccc}
\hline & \multicolumn{3}{c}{ Biochemical pathways } \\
\cline { 2 - 4 } Biomass & EMP & TCA & PPP \\
\hline Above-ground & 0.6008 & 0.8003 & 0.6489 \\
Below-ground & 0.7513 & 0.7230 & 0.7280 \\
\hline
\end{tabular}

EMP, glycolysis pathway; TCA, tricarboxylic acid cycle; PPP, pentose phosphate pathway.

Table III. Correlation coefficients determined by grey correlation analysis between biomass and the content of respiratory substrates and intermediates for Glycyrrhiza uralensis.

\begin{tabular}{lccccc}
\hline & \multicolumn{2}{c}{$\begin{array}{c}\text { Respiratory } \\
\text { substrates }\end{array}$} & & \multicolumn{2}{c}{ Intermediates } \\
\cline { 2 - 3 } \cline { 5 - 6 } Biomass & $\begin{array}{c}\text { Soluble } \\
\text { sugar }\end{array}$ & Starch & $\begin{array}{c}\text { Pyruvic } \\
\text { acid }\end{array}$ & $\begin{array}{c}\text { Acetyl } \\
\text { coenzyme A }\end{array}$ \\
\hline Above-ground & 0.5790 & 0.7377 & & 0.5694 & 0.7350 \\
Below-ground & 0.6110 & 0.5723 & & 0.6363 & 0.6138 \\
\hline
\end{tabular}

Table IV. Correlation coefficients determined by grey correlation analysis between biochemical pathways and the content of intermediates in Glycyrrhiza uralensis.

\begin{tabular}{lccc}
\hline & \multicolumn{3}{c}{ Biochemical pathways } \\
\cline { 2 - 4 } Intermediates & EMP & TCA & PPP \\
\hline Acetyl coenzyme A & 0.5567 & 0.8795 & 0.5841 \\
Pyruvic acid & 0.7287 & 0.5902 & 0.7078 \\
\hline
\end{tabular}

EMP, glycolysis pathway; TCA, tricarboxylic acid cycle; PPP, pentose phosphate pathway.

\section{Discussion}

Root respiration serves an important role in the accumulation of biomass and glycyrrhizic acid in G. uralensis (10). However,
Table V. Correlation coefficients determined by grey correlation analysis between biochemical pathways and the content of glycyrrhizic acid in Glycyrrhiza uralensis.

\begin{tabular}{lc}
\hline Pathway & Correlation with glycyrrhizic acid \\
\hline EMP & 0.6661 \\
TCA & 0.5792 \\
PPP & 0.5839 \\
\hline
\end{tabular}

Table VI. Correlation coefficients determined by grey correlation analysis between intermediates and the content of glycyrrhizic acid in G. uralensis.

\begin{tabular}{lc}
\hline Intermediate & Correlation with glycyrrhizic acid \\
\hline Pyruvic acid & 0.6972 \\
Acetyl coenzyme A & 0.6970 \\
\hline
\end{tabular}

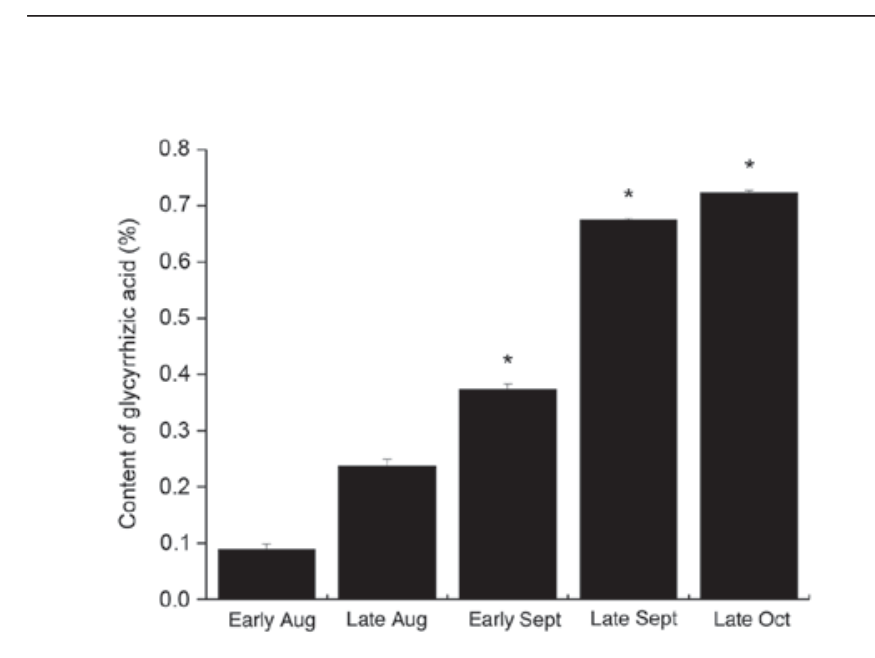

Figure 9. Dynamic changes of the content of glycyrrhizic acid in the root of Glycyrrhiza uralensis. The data were presented as mean \pm standard deviation from at least three experiments. $\mathrm{P}<0.05$, compared with early August.

the effects of respiration pathways on the accumulation levels have not been evaluated. In the present study, the dynamics of root respiration were analyzed, and the correlation between root respiration and the levels of biomass and glycyrrhizic acid in G. uralensis was investigated.

Root respiration is affected by soil temperature, and the respiratory metabolism varies according to the growth stage and plant species (17). In the present study, the respiration rate was measured at $25^{\circ} \mathrm{C}$ in order to exclude the influence 
of temperature (5). No significant difference was observed in the total respiration rate between the analyzed time-points. EMP, TCA and PPP pathways acted concurrently in the root of G. uralensis. Soluble sugar and starch, which are synthesized by photosynthesis, are the substrates of respiration (18). In the present study, the content of soluble sugar and starch increased rapidly, reaching a maximum at early September, followed by a decline. The reason may be that photosynthesis of the above-ground plant was increased prior to September, and the organic substances were mainly used for the growth of the above-ground plant, whereas in and after September, the plant was in a state of dormancy, and the substances were transferred underground. The content of pyruvic acid exhibited a similar trend to the soluble sugar, increasing continuously and then decreasing. The reason may be that pyruvic acid is produced by EMP. The content of acetyl coenzyme A increased initially and then decreased, and reached a maximum in late August, indicating that substance transfer and biosynthesis was the most active in this period.

Root respiration is closely associated with plant biosynthesis. In the present study, it was found that both EMP-TCA and PPP were closely associated with root respiration, which suggests that they may be associated with the biosynthesis and transfer activity in the storage root.

Grey correlation analysis showed that TCA had the greatest degree of correlation (correlation coefficient, 0.8003) with biomass accumulation. The reason may be that the TCA is the most effective process by which plants obtain energy. The results of the present study indicated that starch and acetyl coenzyme A had the closest correlation with above-ground biomass. The reason may be that the aerial parts of the plants had stronger biomass and photosynthetic activity. This could result in increased starch synthesis and a greater degree of active carbon conversion. By contrast, soluble sugar had a lower degree of correlation with above-ground biomass, since the sugar was translated into starch. Grey correlation analysis between biochemical pathways and the intermediates showed that pyruvic acid had the highest degree of correlation with EMP, while acetyl coenzyme A had the highest degree of correlation with TCA. The reason for this may be that pyruvic acid is the product of the EMP pathway, and acetyl coenzyme $\mathrm{A}$ is involved in the TCA directly.

Glycyrrhizic acid is produced from mevalonic acid (MVA) with acetyl coenzyme A as donor (19,20). Acetyl coenzyme $\mathrm{A}$ is produced by a catalytic reaction involving pyruvic acid and is then involved in the TCA (19). In the present study, grey relational analysis showed that pyruvic acid and EMP had a high degree of correlation with glycyrrhizic acid, while acetyl coenzyme A and TCA correlated less strongly with glycyrrhizic acid. This may be due to the fact that TCA occurs in mitochondria, while the MVA pathway occurs in cytoplasm.

In conclusion, among the TCA, PPP and EMP pathways, the TCA pathway exhibited the highest correlation with biomass. Starch and acetyl coenzyme A had the closest association with above-ground biomass. Pyruvic acid and EMP correlated the most strongly with glycyrrhizic acid content. The results of this study may aid the cultivation of G. uralensis. However, the results require verification in further studies.

\section{Acknowledgements}

The present study was supported by grants from the Beijing University of Chinese Medicine (grant no. 2009JYB22-JSO35) and the National Natural Scientific Foundation (grant no. 81573524).

\section{References}

1. Edwards NT, Shugart HH Jr, McLaughlin SB, Harris WF and Reichle DE: Carbon metabolism in terrestrial ecosystems. In: Dynamic Properties Of Forest Ecosystems. Reichle DE (ed). Cambridge University Press, Cambridge, pp499-536, 1980.

2. Zhang Y, Wang J, Liu Y and Wang W: Nitrogen nutrition in Glycyrrhiza uralensis Fisch. J Beijing Forestry University 27: 57-60, 2005 (In Chinese).

3. Sun ZR, Zhai MP, Wang WQ and Li YR: Effects of density on seedling growth and glycyrrhizinic acid content in Glycyrrhiza uralensis. Zhongguo Zhong Yao Za Zhi 32: 2222-22226, 2281, 2007 (In Chinese).

4. Jun-Mai C: Research on adaptation mechanism of Glycyrrhiza uralensis to water deficiency. J Anhui Agricul Sci 16: 080, 2009.

5. Atkin OK, Edwards EJ and Loveys BR: Response of root respiration to changes in temperature and its relevance to global warming. New Phytologist 147: 141-154, 2000.

6. Van Dongen JT, Gupta KJ, Ramírez-Aguilar SJ, Araújo WL, Nunes-Nesi A and Fernie AR: Regulation of respiration in plants: A role for alternative metabolic pathways. J Plant Physiol 168: 1434-1443, 2011.

7. Guo Q, Lv X, Xu F, Zhang Y, Wang J, Lin H and Wu B: Chlorine dioxide treatment decreases respiration and ethylene synthesis in fresh-cut 'Hami'melon fruit. Int J Food Sci Tech 48: 1775-1782, 2013.

8. Vanlerberghe GC: Alternative oxidase: A mitochondrial respiratory pathway to maintain metabolic and signaling homeostasis during abiotic and biotic stress in plants. Int J Mol Sci 14: 6805-6847, 2013.

9. Högberg P, Nordgren A and Ågren GI: Carbon allocation between tree root growth and root respiration in boreal pine forest. Oecologia 132: 579-581, 2002.

10. Guo P, Sun Z, Liu W, Chen L, Du Y and Wei X: Correlation analysis between the rate of respiration in the root and the active components in licorice (Glycyrrhiza uralensis). Exp Ther Med 7: 270-274, 2014.

11. Lee MS, Nakane K, Nakatsubo T and Koizumi H: Seasonal changes in the contribution of root respiration to total soil respiration in a cool-temperate deciduous forest. In: Roots: The Dynamic Interface between Plants and the Earth. Abe J (ed). Springer, Berlin, pp311-318, 2003.

12. Buysse J and Merckx R: An improved colorimetric method to quantify sugar content of plant tissue. J Exp Botany 44: $1627-1629,1993$.

13. Johnson G and Scholes G: Micro-determination of acetaldehyde as its 2:4-dinitrophenylhydrazone. Analyst 79: 217-279, 1954 .

14. Brown AL and Wray JL: Correlated changes of some enzyme activities and cofactor and substrate contents of pea cotyledon tissue during germination. Biochem J 108: 437-444, 1968.

15. Zhang Q and Ye M: Chemical analysis of the Chinese herbal medicine Gan-Cao (licorice). J Chromatogr A 1216: 1954-1969, 2009.

16. Dai J, Liu X and Hu F: Research and application for grey relational analysis in multigranularity based on normality grey number. ScientificWorldJournal 2014: 312645, 2014.

17. Zogg GP, Zak DR, Burton AJ and Pregitzer KS: Fine root respiration in northern hardwood forests in relation to temperature and nitrogen availability. Tree Physiol 16: 719-725, 1996.

18. Goldschmidt EE and Huber SC: Regulation of photosynthesis by end-product accumulation in leaves of plants storing starch, sucrose, and hexose sugars. Plant Physiol 99: 1443-1448, 1992.

19. Lichtenthaler HK: The 1-deoxy-D-xylulose-5-phosphate pathway of isoprenoid biosynthesis in plants. Annu Rev Plant Physiol Plant Mol Biol 50: 47-65, 1999.

20. Chappell J, Wolf F, Proulx J, Cuellar R and Saunders C: Is the reaction catalyzed by 3-hydroxy-3-methylglutaryl coenzyme a reductase a rate-limiting step for isoprenoid biosynthesis in plants? Plant Physiol 109: 1337-1343, 1995. 